\title{
Quantum Degeneracy in Atomic Point Contacts Revealed by Chemical Force and Conductance
}

\author{
Yoshiaki Sugimoto, ${ }^{1, *}$ Martin Ondráček, ${ }^{2}$ Masayuki Abe,${ }^{3}$ Pablo Pou, ${ }^{4}$ Seizo Morita, ${ }^{1}$ Ruben Perez, ${ }^{4}$ \\ Fernando Flores, ${ }^{4}$ and Pavel Jelínek ${ }^{2, \dagger}$ \\ ${ }^{1}$ Graduate School of Engineering, Osaka University, 2-1 Yamada-Oka, 565-0871 Suita, Osaka, Japan \\ ${ }^{2}$ Institute of Physics, Academy of Sciences of the Czech Republic, Cukrovarnická 10, 16200 Prague, Czech Republic \\ ${ }^{3}$ Graduate School of Engineering, Nagoya University, Furo-cho, Chikusa-ku, Nagoya 464-8603, Japan \\ ${ }^{4}$ Departamento de Física Teórica de la Materia Condensada and Condensed Matter Physics Center (IFIMAC), \\ Universidad Autónoma de Madrid, 28049 Madrid, Spain
}

(Received 31 May 2013; published 5 September 2013)

\begin{abstract}
Quantum degeneracy is an important concept in quantum mechanics with large implications to many processes in condensed matter. Here, we show the consequences of electron energy level degeneracy on the conductance and the chemical force between two bodies at the atomic scale. We propose a novel way in which a scanning probe microscope can detect the presence of degenerate states in atomic-sized contacts even at room temperature. The tunneling conductance $G$ and chemical binding force $F$ between two bodies both tend to decay exponentially with distance in a certain distance range, usually maintaining direct proportionality $G \propto F$. However, we show that a square relation $G \propto F^{2}$ arises as a consequence of quantum degeneracy between the interacting frontier states of the scanning tip and a surface atom. We demonstrate this phenomenon on the $\mathrm{Si}(111)-(7 \times 7)$ surface reconstruction where the $\mathrm{Si}$ adatom possesses a strongly localized dangling-bond state at the Fermi level.
\end{abstract}

PACS numbers: 73.20.At, 68.37.Ef, 68.37.Ps

The invention of scanning probe microscopy (SPM), almost three decades ago, boosted research of matter at the nanoscale. Nowadays, SPM techniques are routinely used not only for imaging with unprecedented atomic resolution [1,2] or single atom manipulation [3] but also for an advanced characterization on an atomic scale [4-9]. What is more, SPM provides direct access to the nanoscale where the quantum phenomena tend to emerge. Many of them have been the subject of intensive SPM studies [10,11]. Nevertheless, quantum degeneracy, one of the basic concepts of quantum mechanics, has received little attention so far. The quantum degeneracy is defined as the presence of two or more quantum states localized at the same energy level. Among others, the degeneracy plays an important role in the low temperature charge transport phenomena at the nanoscale, such as the Kondo effect modulated by mechanical action [12], resonant tunneling [13], or Coulomb blockade [14]. Recent progress in scanning probe instrumentation has allowed the combination of atomic force microscopy (AFM) and scanning tunneling microscopy (STM) [15] where both tunneling current and force are collected simultaneously. This approach opens new possibilities not only in the characterization at the atomic scale but also to understand the fundamental relation between the chemical force and the conductance in a well-defined atomic contact [see Fig. 1(a)]. In this Letter, we demonstrate that the quantum degeneracy of frontier electronic states of two interacting bodies forming an atomic point contact can be explored by precise AFM/STM experiments, even at room temperature, through an intimate relation between the chemical force and the conductance.
The understanding of the mechanical and transport properties at the atomic scale is of importance not only in fundamental physics but also for practical applications in nanotechnology. An atomic scale junction forms when two objects (e.g., scanning probe and sample) are brought near to mechanical contact with a subnanometer distance control. In a far distance regime, where electron wave functions do not overlap, the interaction between two bodies is driven by longrange van der Waals or electrostatic forces [16]. As two objects are brought near to the contact, the overlap of their wave functions gives rise to a short-range force $F$, which is a signature of chemical bond formation between the outermost atoms of the tip and sample. It has been demonstrated both theoretically [17] and experimentally [16] that the chemical force $F$ follows an exponential dependence $F \propto \exp \left(-\kappa_{F} z\right)$ on distance $z$, where $\kappa_{F}$ is the characteristic decay constant. Simultaneously, the wave function overlap enables the tunneling current $\left(I_{t}\right)$ to flow through the atomic junction when a bias voltage $\left(V_{s}\right)$ is applied. In general, the conductance $(G=$ $I_{t} / V_{s}$ ) also obeys the exponential dependence $G \propto$ $\exp \left(-\kappa_{G} z\right)$ on distance $z$ in the tunneling regime [18]. Here, the characteristic decay coefficient $\kappa_{G}$ is related to the work function $\Phi$ as follows: $\kappa_{G}=1.025 \sqrt{\Phi}$ (where $\kappa_{G}$ is in $\AA^{-1}$ and $\Phi$ in $\mathrm{eV}$ ). To sum up, both the chemical interaction force $F$ and the conductance $G$ show a very similar, namely, exponential, characteristic dependence on the tip-sample distance [18].

Based on this fact, Chen [18] derived a simple rule postulating the conductance to be proportional to the square of the chemical force $\left(G \propto F^{2}\right)$. Later, supported by the density functional theory (DFT) simulations [19], Hofer and Fischer 


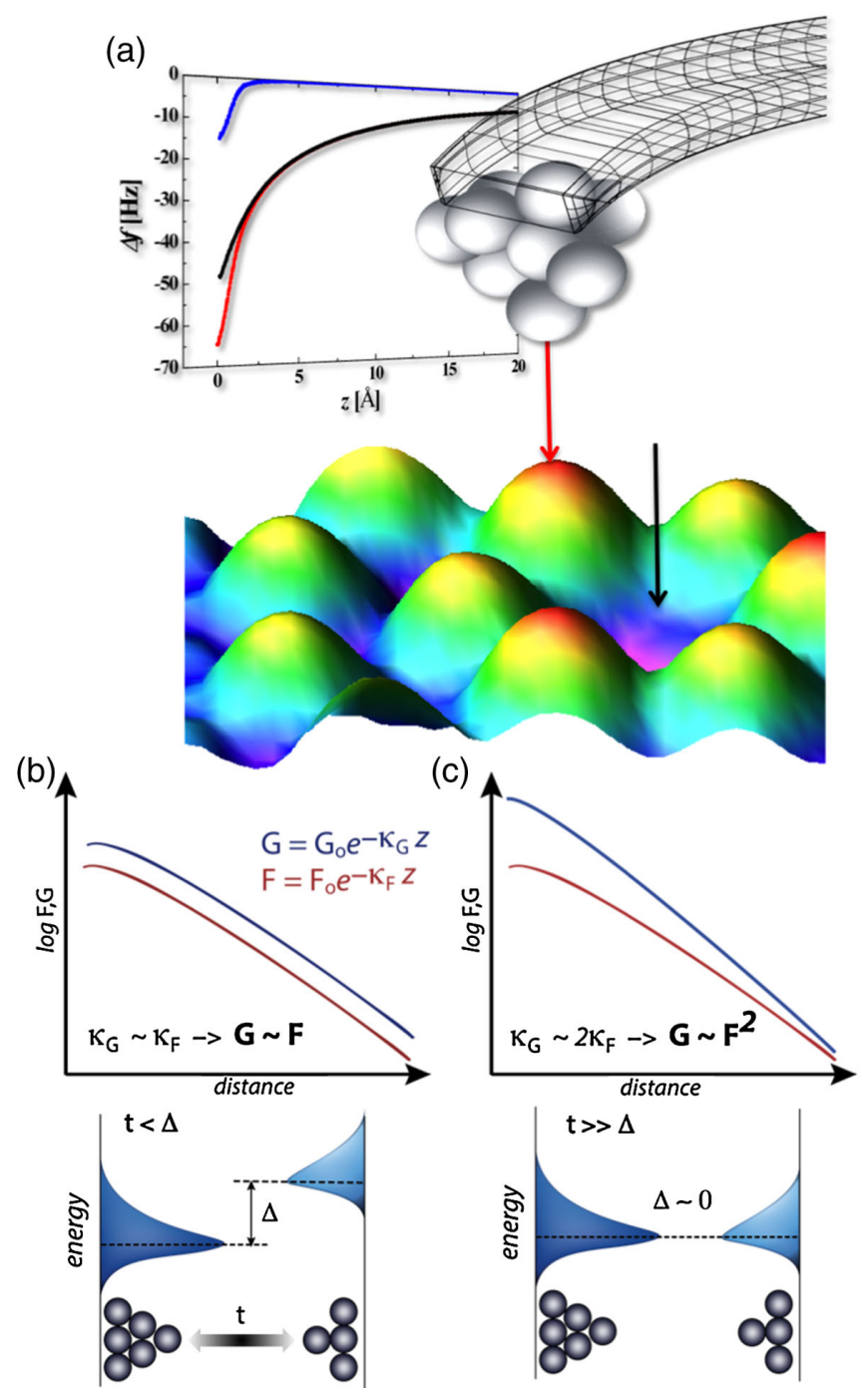

FIG. 1 (color online). AFM/STM sensor interacting with a sample. (a) Schematic view of a simultaneous AFM/STM measurement using an oscillating sensor on the $\operatorname{Si}(111)-(7 \times 7)$ surface. Subtraction of $\Delta f(z)$ curves acquired over the adatom (red or light gray curve) and hollow (black curve) positions allows precise estimation of the short-range component (blue or dark gray curve). For more details about the extraction of the short-range force, see Ref. [28]. (b),(c) The relation between the short-range force $F$ and the tunneling conductance $G$ in the (b) nondegenerate and (c) degenerate cases. The splitting of the energy levels leading to the presence or absence of degeneracy is sketched below the plots in (b) and (c).

proposed a different rule, a linear relation between the quantities $(G \propto F)$. The existence of two concurrent relations initiated a long-standing debate, including both experiment [20-23] and theory [21,24].

Recently, we introduced a generalized theoretical model [25], which rationalizes the coexistence of the two characteristic scaling regimes discussed above. The interaction energy between two states with similar broadening [see Figs. 1(b) and 1(c)] can be written as $E \propto \sqrt{t^{2}+\Delta^{2}}$, where $\Delta$ and $t$ represent, respectively, the energy degeneracy and hopping between the frontier electronic levels involved in the interaction process. Two limiting cases may arise, depending on the ratio between the energy splitting $\Delta$ and the hopping element $t$. In the case of $t<\Delta$ [see Fig. 1(b)], the interaction energy can be recast as $E \propto t^{2}$. In the opposite limit $t \gg \Delta$ [see Fig. 1(c)], the interaction energy tends to be proportional to the hopping, i.e., $E \propto t$. On the other hand, according to the Bardeen formalism [18], the conductance is proportional to the square of the hopping elements, i.e., $G \propto t^{2}$.

From the discussion above, we immediately see the origin of the two different relations between the conductance and the chemical force. The interaction between two nondegenerate and/or delocalized electronic states renders the linear relation $G \propto F$ [19]. The existence of this regime has been unambiguously confirmed by means of AFM/ STM measurements supported by first principles calculations with nonperturbative treatment of the electron transport in metallic atomic point contacts [21]. On the other hand, the square-rule regime $G \propto F^{2}$ prevails in the case of quantum degeneracy; i.e., the interaction occurs between two strongly localized and degenerate electronic states, giving $t \gg \Delta$. However, the conclusive affirmation of the square relation $G \propto F^{2}$ has been missing so far.

In this Letter, we combine simultaneous force or current measurements by means of SPM and DFT calculations on the $\operatorname{Si}(111)-(7 \times 7)$ surface to demonstrate the validity of the square relation $G \propto F^{2}$ between the conductance and the chemical force. Furthermore, we will demonstrate that the presence of this regime is unequivocally related to the quantum degeneracy of the frontier electronic states involved.

For this purpose, we have deliberately chosen the $\mathrm{Si}(111)-(7 \times 7)$ surface to ensure the presence of localized states on both the sample and tip. On this surface reconstruction, each $\mathrm{Si}$ adatom possesses a localized and partially occupied [26,27] dangling-bond state at the Fermi level in contrast to strongly delocalized states on metal surfaces [21]; see a comparison of the electron density distribution for the $\mathrm{Si}(111)-(7 \times 7)$ and $\mathrm{Cu} / \mathrm{Cu}(111)$ surfaces in Fig. S1 of Ref. [28]. Second, in the experiments, controlled gentle landing of metallic tips on the $\mathrm{Si}(111)-(7 \times 7)$ is mandatory to achieve atomic resolution in both AFM and STM modes. The enhanced resolution is a consequence of the formation of a Si cluster on the tip apex [29]. Extensive theoretical studies of Si tips [30] indicate that the most probable atomic structure of the tip apex consists of the outermost $\mathrm{Si}$ atom with a dangling bond. Consequently, the interaction during a tip approach is controlled by two dangling-bond states located on the frontier $\mathrm{Si}$ atoms of the tip and sample, respectively.

We have performed calculations of the AFM force based on DFT, followed by transport calculations of the tunneling current in the Green's function formalism. We employ the plane-wave-based code VASP [31] with Vanderbilt ultrasoft pseudopotentials [32] and the Perdew-Wang 91 version [33] of the generalized gradient approximation to calculate the short-range forces. We make use of well-established models of Si tips [30] placed over a corner adatom of a $7 \times 7$ supercell on the reconstructed $\operatorname{Si}(111)$ surface. The 
tip was gradually approached towards the $\mathrm{Si}$ adatom by $0.25 \AA$, and the atomic structure was relaxed at each step until the forces acting on free atoms were below $1 \mathrm{meV} / \AA$. The tunneling conductance was calculated within a Green's function formalism [34], as implemented in the FIREBALL code [35]. The geometry of the system for the electron transport was taken from the VASP calculations. More details are given in Ref. [28].

Our theoretical results (Fig. 2) using a Si-tip model show that both the force $F$ and the conductance $G$ follow the exponential law in the far distance regime, until $z \approx 5.25 \AA$ (see the linear progression on the $\log$ plots in the insets of Fig. 2). In a close distance regime $(z<5.25 \AA)$, both the chemical force and the conductance substantially deviate from the exponential relation. An increase of the force is accompanied by a strong reduction of the conductance. This phenomenon is related to a modification of the electronic structure of the surface adatom due to the formation of the chemical bond [36]. In the far distance range, where the exponential decay holds, the characteristic decay constants for the conductance and the chemical force are $\kappa_{G}=$ $22.2 \mathrm{~nm}^{-1}$ and $\kappa_{F}=11.3 \mathrm{~nm}^{-1}$, respectively. The ratio $\kappa_{G} / \kappa_{F}$ between them is $\approx 1.96$, very close to the factor 2 . Therefore, we can conclude that the square relation $G \propto F^{2}$ holds between the conductance and the chemical force. This observation is in contrast to previous atomic metal junction studies [20,21], where $F$ and $G$ were found to be proportional.

According to the theoretical analysis [25], the square relation $G \propto F^{2}$ occurs when the hopping interaction $t$ between frontier orbitals is much larger than their energy

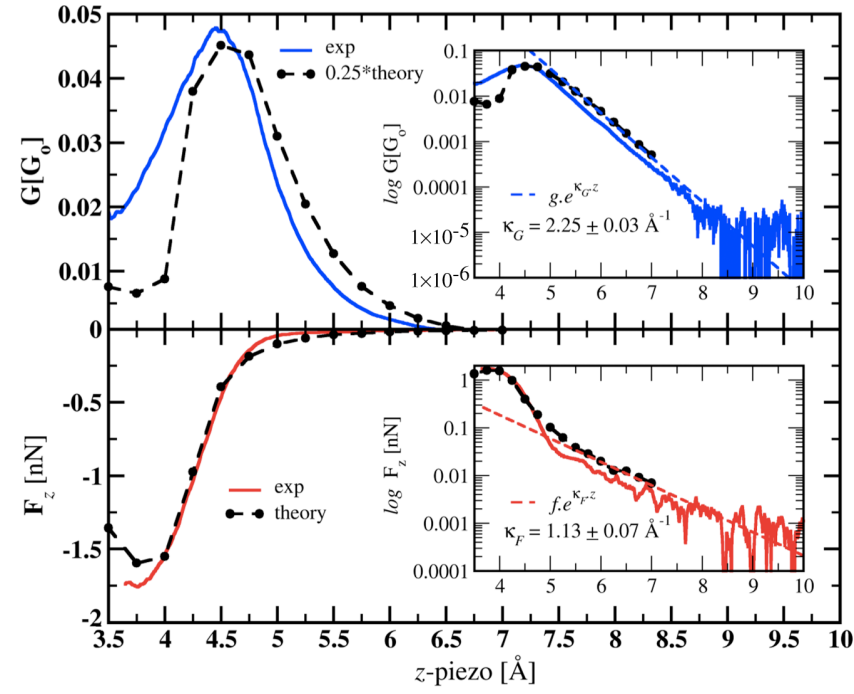

FIG. 2 (color online). Tunneling conductance and short-range force component. Top: Comparison between calculated (dashed black line with symbols) and measured (thin blue solid line) tunneling conductances $G$ as a function of tip-surface separation z. Bottom: Calculated (dashed black line with symbols) and measured (thin solid red line) $z$ dependences of the short-range force $F$. Logarithmic plots with estimated decay constants are shown in the insets. difference $\Delta$. In Fig. 3, we plot the projected density of states (PDOS) on both the Si adatom and the Si apex atom in the far distance regime. At these distances, the PDOS remains practically intact, exhibiting two nearly degenerate frontier states close to the Fermi level with the same energy width, one on the tip apex and the other on the surface adatom. Taking advantage of the local basis set formalism used in the transport calculations, the key parameters of our model [25] — the energy splitting $\Delta$ and hopping $t$ - can be directly estimated. In this particular case, we obtain the following values: $\Delta=5 \mathrm{meV}$ and $t=75 \mathrm{meV}$ at distance $z=6 \AA$. Using these numbers, we obtain $\sqrt{t^{2}+\Delta^{2}} \approx$ $(75.2 \mathrm{meV}) \approx t$, so the relation $E \propto t$ holds, which immediately implies the presence of the quadratic regime $G \propto$ $F^{2}$, the fingerprint of the quantum degeneracy.

To confirm our theoretical findings, we performed precise simultaneous AFM/STM measurements on the $\operatorname{Si}(111)-(7 \times 7)$ surface. The simultaneously acquired frequency shift $\Delta f_{\mathrm{SR}}(z)$ corresponding to the chemical force and the averaged tunneling current $\left\langle I_{t}(z)\right\rangle$ above the Si adatom are shown in Fig. 4(a). The experiments were carried out using a custom-built ultrahigh vacuum AFM/STM operated at room temperature. Commercial Pt-Ir coated Si cantilevers (NCLPt, NanoWorld) were cleaned by Ar-ion sputtering before use. After that, the tip was intentionally poked to the surface until atomic resolution was obtained in both the AFM and STM modes. A cantilever deflection sensor based on an optical interferometer enabled force measurements with high sensitivity and guaranteed decoupling of the force and tunneling current signals. The interaction force was measured by the frequency modulation detection method. To obtain only the short-range component $\Delta f_{\mathrm{SR}}(z)$, we subtracted the frequency shift $\Delta f(z)$ curves acquired over the adatom and the hollow site [7], as shown in Fig. 1(a). For more details, see Ref. [28]. $\Delta f_{\mathrm{SR}}(z)$ was converted into $F(z)$ using the Sader

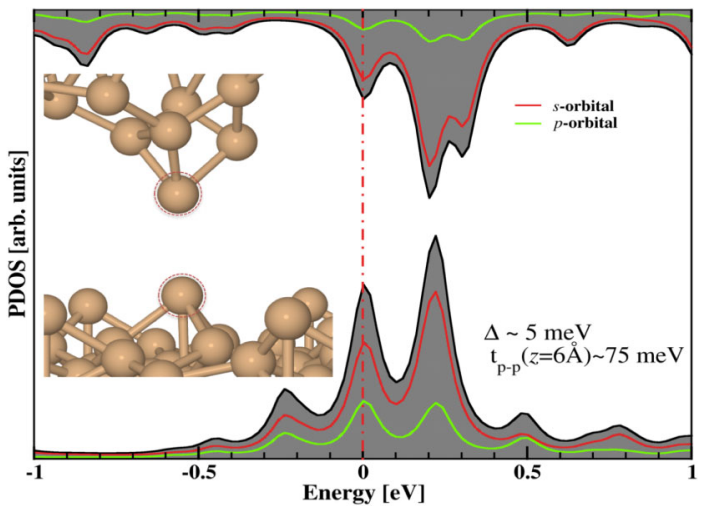

FIG. 3 (color online). Electron states on the tip and on the surface. Density of states (PDOS) projected on a corner adatom of the $\mathrm{Si}(111)-(7 \times 7)$ surface (bottom plot) and on the outermost apex atom of the Si tip (top plot, turned upside-down). Black curves and shaded areas, complete PDOS; red curves, $s$-orbital contribution to PDOS; green curves, $p$-orbital contribution to PDOS. Inset: Ball-and-stick model of the SPM tip apex above the surface adatom. 

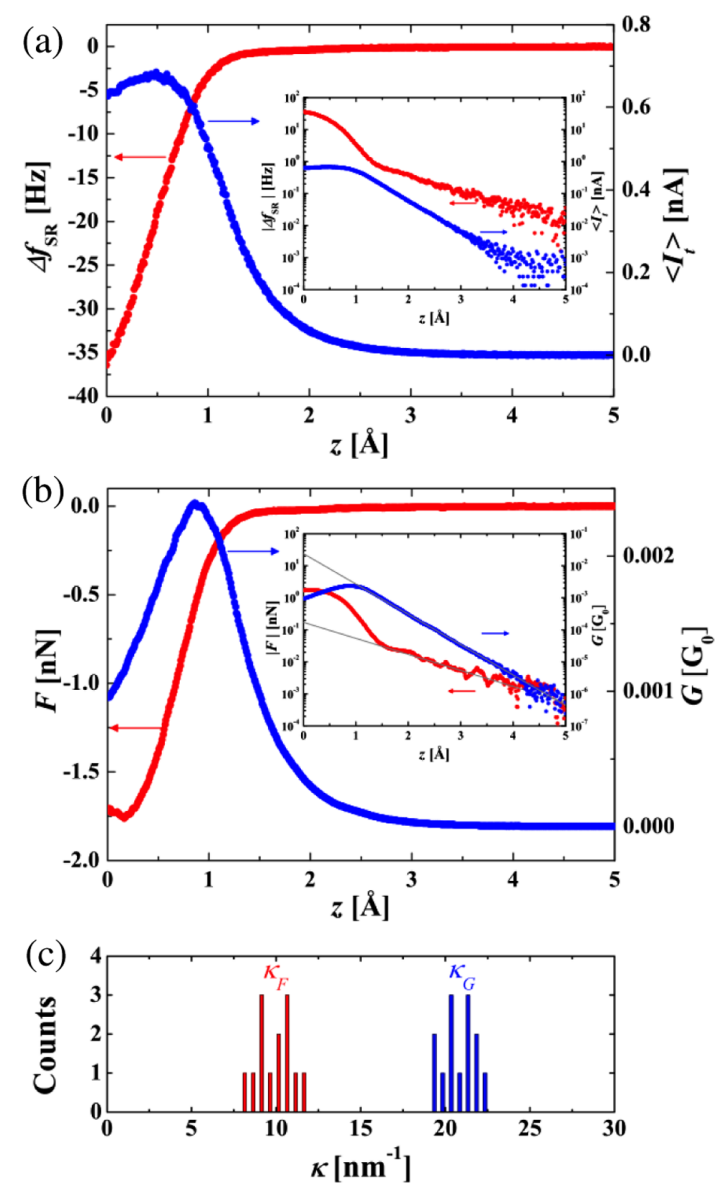

FIG. 4 (color online). Results of force and conductance measurements. (a) $\Delta f_{\mathrm{SR}}(z)$ (red or light gray line) and $\left\langle I_{t}(z)\right\rangle$ (blue or dark gray line) curves acquired above the corner adatom of the $\operatorname{Si}(111)-(7 \times 7)$ surface. $\Delta f_{\mathrm{SR}}(z)$ was obtained by subtraction of the long-range force contribution estimated from the curve above the corner hole. (b) $F(z)$ (red or light gray line) and $G(z)$ (blue or dark gray line) curves derived from (a) using the conversion formulas. Here, $G_{0}=2 e^{2} / h=(12906 \Omega)^{-1}$. Logarithmic plots are shown in the insets in (a) and (b), respectively. The exponential fitting curves into $F(z)$ and $G(z)$ for $z>5.25 \AA$ are also shown in the inset of (b). The acquisition parameters were $f_{0}=$ $144.4074 \mathrm{kHz}, A=123 \AA, k=19.8 \mathrm{~N} / \mathrm{m}, Q=27000$, and $V_{s}=+100 \mathrm{mV}$. The imaging set point for dynamic STM was $\left\langle I_{t}\right\rangle=20 \mathrm{pA}$. The tip-sample distance, denoted as $z$, in (a) and (b) is aligned according to the DFT calculations (see Fig. 2). (c) Histogram of the magnitudes of decay parameters $\kappa_{F}$ and $\kappa_{G}$ which were acquired using 16 different tips with 8 different cantilevers.

formula [37], while $\left\langle I_{t}(z)\right\rangle$ was also converted into the instantaneous tunneling current $\left[I_{t}(z)\right]$ by a complementary formula [38]. The resulting chemical force $F(z)$ and the conductance calculated as $G(z)=I_{t}(z) / V_{s}$ are shown in Fig. 4(b). For more details on the experimental conditions, see Ref. [28].

The experimental data show very similar tendencies, as the theoretical set (see the comparison in Fig. 2), both the chemical force $F(z)$ and the conductance $G(z)$, obey the exponential law in the same distance regime $(z>$ $5.25 \AA$ ) with very similar characteristic decay constants:
$\kappa_{F}=10.53 \pm 0.20 \mathrm{~nm}^{-1}$ and $\kappa_{G}=21.79 \pm 0.04 \mathrm{~nm}^{-1}$, respectively. The ratio $\kappa_{G} / \kappa_{F} \approx 2.07 \pm 0.04$ is again very close to the factor of 2 , which points out the presence of the quantum degeneracy.

We should stress that very similar scaling between $G$ and $F$ was observed repeatedly, using different tips and/or cantilevers (see Ref. [28], Fig. S3). In particular, the characteristic decay constants $\kappa_{F}$ and $\kappa_{G}$ recorded in the far distance regime were well reproducible [see Fig. 4(c)], maintaining the square relation $\left(G \propto F^{2}\right)$. On the other hand, we noticed a strong variation of the prefactor in the exponential dependence of the conductance by an order of magnitude between experimental sessions. We attribute this phenomenon to modifications of the mesoscopic structure of the probe, which consists of the formation of small Si clusters on the tip. The presence of Si nanoclusters might strongly influence the charge transport in the probe, as has been already shown [29]. Nevertheless, the characteristic decay constant $\kappa_{G}$ remains almost unchanged (cf. Ref. [28], Figs. S3 and S4).

Let us also note that we have also detected the $G \propto F^{2}$ regime in our measurements, applying bias voltages up to a few hundreds of $\mathrm{mV}$. In principle, energy level shifts of that order should break the degeneracy, yielding the lineal $G \propto$ $F$ relation. However, the presence of a significant on-site charge interaction on the localized states screens out the external field $V_{s}$ [39], a mechanism associated with some charge transfer between the dangling bonds and reduction of the energy level shift by an order of magnitude with respect to the applied voltages. Consequently, the screening keeps the degeneracy on this system up to voltages of at least $0.5 \mathrm{~V}$ (for more detailed discussion, see Ref. [28]).

Here, we showed that simultaneous AFM/STM spectroscopy supported by theoretical calculations provides new insight into dependences between fundamental quantities at the atomic scale. Namely, the analysis of the chemical force and the conductance reveals an intimate relation between these two quantities and the importance of the quantum degeneracy for the relation. While the characteristic decay of the conductance $\kappa_{G}$ is mostly unaffected by the presence of quantum degeneracy, the decay of the shortrange force $\kappa_{F}$ is dramatically reduced, namely, by a factor of 2. Consequently, a correct description of the interaction between two bodies mediated by strongly localized electronic states (e.g., dangling-bond states on a semiconductor surface) using pairwise classical potentials (e.g., the Morse potential [40]) requires the proper correction of the characteristic decay when the quantum degeneracy takes place. On the other hand, for materials with rather delocalized electronic states, such as simple or noble metals [21], the characteristic decay of the interaction can be, in principle, determined directly from STM measurements via estimation of the characteristic decay length $\kappa_{G}$ because $\kappa_{G} \approx \kappa_{F}$.

In summary, we have showed that the quantum degeneracy phenomena can be detected by SPM even at room temperature via the intimate relation between the chemical force and the conductance. In particular, the relation between the chemical force $F$ and the conductance $G$ in 
atomic scale contacts, where the interaction takes place between two nearly degenerate spatially and energetically localized states, becomes $G \propto F^{2}$ in the far distance regime. This observation can be used not only as a measure of the quantum degeneracy of two interacting states but it should also be taken into account in designing empirical pairwise potentials, where the characteristic decay constant $\kappa_{F}$ should be modified accordingly.

This work was supported by Grants-in-Aid for Scientific Research (Grants No. 22221006, No. 24360016, No. 24651116, No. 22760028, and No. 25106002) from the Ministry of Education, Culture, Sports, Science, and Technology of Japan (MEXT), Funding Program for Next Generation World-Leading Researchers. P. J. and M. O. acknowledge the financial support of GAAV M100101207. M. O. acknowledges the support provided by the Czech Science Foundation (GAČR) under Project No. P204/11/P578. R. P. and P.P. acknowledge Projects No. MAT2011-23627, No. CSD2010-00024, No. PLE2009-0061 (MINECO, Spain), and CAM S2009/ MAT-1467. P.P. was supported by the Ramón y Cajal Program (MINECO, Spain).

*sugimoto@afm.eei.eng.osaka-u.ac.jp

†jelinekp@fzu.cz

[1] L. Gross, F. Mohn, N. Moll, P. Liljeroth, and G. Meyer, Science 325, 1110 (2009).

[2] J. Welker and F. Giessibl, Science 336, 444 (2012).

[3] D. M. Eigler and E. K. Schweizer, Nature (London) 344, 524 (1990).

[4] R. Wiesendanger, Rev. Mod. Phys. 81, 1495 (2009).

[5] B. Stipe, M. Rezaei, and W. Ho, Science 280, 1732 (1998).

[6] J. Repp, G. Meyer, F. Olsson, and M. Persson, Science 305, 493 (2004).

[7] M. A. Lantz, H. J. Hug, R. Hoffmann, P. J. A. van Schendel, P. Kappenberger, S. Martin, A. Baratoff, and H. J. Güntherodt, Science 291, 2580 (2001).

[8] Y. Sugimoto, P. Pou, M. Abe, P. Jelínek, R. Pérez, S. Morita, and O. Custance, Nature (London) 446, 64 (2007).

[9] C. F. Hirjibehedin, C.-Y.Lin, A. F. Otte, M. Ternes, C. P. Lutz, B. A. Jones, and A. J. Heinrich, Science 317, 1199 (2007).

[10] M.F. Crommie, C. P. Lutz, and D. M. Eigler, Nature (London) 363, 524 (1993).

[11] H. C. Manoharan, C.P. Lutz, and D. M. Eigler, Nature (London) 403, 512 (2000).

[12] J. J. Parks, A. R. Champagne, T. A. Costi, W. W. Shum, A. N. Pasupathy, E. Neuscamman, S. Flores-Torres, P. S. Cornaglia, A. A. Aligia, and C. A. Balseiro et al., Science 328, 1370 (2010).

[13] R. Tsu and L. Esaki, Appl. Phys. Lett. 22, 562 (1973).
[14] K. Ono, D. Austing, Y. Tokura, and S. Tarucha, Science 297, 1313 (2002).

[15] F. J. Giessibl, Appl. Phys. Lett. 73, 3956 (1998).

[16] U. Dürig, O. Züger, and D. W. Pohl, Phys. Rev. Lett. 65, 349 (1990).

[17] J. H. Rose, J. Ferrante, and J. R. Smith, Phys. Rev. Lett. 47, 675 (1981).

[18] C.J. Chen, Introduction to Scanning Tunneling Microscopy (Oxford University, New York, 2008), 2nd ed.

[19] W. A. Hofer and A. J. Fisher, Phys. Rev. Lett. 91, 036803 (2003).

[20] G. Rubio-Bollinger, P. Joyez, and N. Agraït, Phys. Rev. Lett. 93, 116803 (2004).

[21] M. Ternes, C. González, C. P. Lutz, P. Hapala, F. J. Giessibl, P. Jelínek, and A. J. Heinrich, Phys. Rev. Lett. 106, 016802 (2011).

[22] C. Loppacher, M. Bammerlin, M. Guggisberg, S. Schär, R. Bennewitz, A. Baratoff, E. Meyer, and H.-J. Güntherodt, Phys. Rev. B 62, 16944 (2000).

[23] Y. Sun, H. Mortensen, S. Schär, A.-S. Lucier, Y. Miyahara, P. Grütter, and W. Hofer, Phys. Rev. B 71, 193407 (2005).

[24] C. J. Chen, Phys. Rev. Lett. 96, 069701 (2006).

[25] P. Jelínek, M. Ondráček, and F. Flores, J. Phys. Condens. Matter 24, 084001 (2012).

[26] J. Ortega, F. Flores, and A. LevyYeyati, Phys. Rev. B 58, 4584 (1998).

[27] R. Losio, K. N. Altmann, and F. J. Himpsel, Phys. Rev. B 61, 10845 (2000).

[28] See Supplemental Material at http://link.aps.org/ supplemental/10.1103/PhysRevLett.111.106803 for more details.

[29] K. Morita, Y. Sugimoto, M. Abe, and S. Morita, Appl. Phys. Express 4, 115201 (2011).

[30] P. Pou, S. A. Ghasemi, P. Jelinek, T. Lenosky, S. Goedecker, and R. Perez, Nanotechnology 20, 264015 (2009).

[31] G. Kresse and J. Furthmüller, Phys. Rev. B 54, 11169 (1996).

[32] D. Vanderbilt, Phys. Rev. B 41, 7892 (1990).

[33] J. P. Perdew, J. A. Chevary, S. H. Vosko, K. A. Jackson, M. R. Pederson, D. J. Singh, and C. Fiolhais, Phys. Rev. B 46, 6671 (1992).

[34] J. M. Blanco, F. Flores, and R. Pérez, Prog. Surf. Sci. 81, 403 (2006).

[35] J. Lewis, P. Jelínek, J. Ortega, A. A. Demkov, D. G. Trabada, B. Haycock, H. Wang, G. Adams, J. K. Tomfohr, and E. Abad et al., Phys. Status Solidi B 248, 1989 (2011).

[36] P. Jelínek, M. Švec, P. Pou, R. Perez, and V. Cháb, Phys. Rev. Lett. 101, 176101 (2008).

[37] J. E. Sader and S. P. Jarvis, Appl. Phys. Lett. 84, 1801 (2004).

[38] J. E. Sader and Y. Sugimoto, Appl. Phys. Lett. 97, 043502 (2010).

[39] E. Abad, C. González, J. Ortega, and F. Flores, Org. Electron. 11, 332 (2010).

[40] P. M. Morse, Phys. Rev. 34, 57 (1929). 